\title{
Interleukin-37: A Peacekeeper at the Intestinal Borders
}

\author{
Luca Pastorelli $^{1,2,3}$ - Theresa T. Pizarro ${ }^{1}$
}

Published online: 13 March 2017

(c) Springer Science+Business Media New York 2017

\section{Introduction}

The interleukin (IL)-1 family of cytokines encompasses a wide spectrum of soluble mediators and receptor antagonists. Their expression, present in nearly all organ systems, is of particular importance at mucosal barriers interfacing the external microenvironment, such as the skin, the respiratory tract, and the gastrointestinal (GI) system. IL-1 and IL-1-related cytokines are key factors in the promotion, enhancement, and regulation of host innate immunity and in the orchestration of their interplay with the adaptive immune system. Interestingly, several IL-1 family members, including IL-1 $\alpha / \mathrm{IL}-1 \beta$, IL-18, and IL-33, display dichotomous, opposing functions in the pathogenesis of chronic intestinal inflammation, by both inducing inflammation and promoting epithelial restitution/repair and mucosal healing [1]. Emerging evidence, however, suggests that the IL-1 family member 7 (IL-1F7), also termed IL-37, possesses predominant and seemingly exclusive anti-inflammatory activities, particularly in the setting of inflammatory bowel disease (IBD) and other colitic conditions.

Theresa T. Pizarro

theresa.pizarro@case.edu

Luca Pastorelli

luca.pastorelli@unimi.it

1 Department of Pathology, Case Western Reserve University School of Medicine, Cleveland, OH 44106, USA

2 Department of Biomedical Sciences for Health, University of Milan, 20133 Milan, Italy

3 Gastroenterology and Digestive Endoscopy Unit, IRCCS Policlinico San Donato, 20097 San Donato Milanese, Italy

\section{Discovery and Characterization of IL-37}

Although the existence of IL-1F7 was discovered in 2000 by in silico studies, the mediator only began to be characterized in terms of its structure, function, and biology a decade later and was subsequently named IL-37 [2]. Seminal studies clearly reported that IL-37 dampens both innate and adaptive immune responses, thus reducing general inflammation; indeed, IL-37, when present during Toll-like/IL-1 receptor stimulation or uric acid sensing, greatly reduces the synthesis of many proinflammatory cytokines [2,3]. Consistent with these findings, experiments utilizing several different animal models support the concept that IL-37 is protective toward inflammatory conditions, such as endotoxic shock, myocardial infarction, metabolic syndrome, asthma, acute lung injury, and ischemic liver disease [3].

With regard to chronic inflammatory conditions of the GI tract, IL-37 reduces the severity of dextran sulfate sodium (DSS)-induced colitis in mice through decreased colonic production of IL-1 $\beta$ and tumor necrosis factor (TNF), increased IL-10 secretion, and an overall reduction in the number of $\mathrm{CD}^{+} \mathrm{IFN}^{+} \mathrm{T}$ lymphocytes $[4,5]$. Interestingly, experiments using this model of acute colitis indicate that, from a functional point of view, the most important cellular sources of IL-37 are of hematopoietic and mesenchymal origin [4]. Of note, however, is that mice appear to lack the IL-37 gene [3]. As such, experiments utilizing mouse models of disease, including experimental colitides, have been conducted using IL-37 transgenic mice, genetically engineered to express human IL-37 [4], or normal rodents injected with mesenchymal stromal cells transfected with the human IL-37 gene [5]. Indeed, while these studies provide important insight into IL-37 function, these results should be interpreted with caution, bearing in 
mind that alternative cellular sources of IL-37 may also contribute substantially to its immunoregulatory activities in the GI tract.

\section{Concepts and Controversies Surrounding the Function of IL-37 During Chronic Intestinal Inflammation}

The study from Gunaltay et al. [6], published in the current issue of Digestive Diseases and Sciences, explores the possibility that colonic epithelial-derived IL-37 promotes the maintenance of intestinal immune homeostasis through the downregulation of gut proinflammatory molecules. Using CRISPR/Cas9 technology in a human colonic epithelial cell line, the authors demonstrate that even a modest reduction in IL-37 expression augments production of chemokines following activation of the Toll-like receptor (TLR)5 signaling pathway after exposure to bacterial-derived flagellin. These findings are notable since they once again emphasize the central importance of the intestinal epithelium in contributing to the regulation of both innate and adaptive immune responses triggered by the gut microbiome [7]. Indeed, the intestinal epithelium is constantly barraged by components of commensal and pathogenic bacteria, as well as by other potentially harmful insults. Depending on the immunological status and genetic predisposition of the host, intestinal epithelial cells respond by producing several proinflammatory molecules, including not only IL- $1 \alpha$, IL-18, and IL-8, and other chemotactic factors [7], but also anti-inflammatory molecules (e.g., IL1Ra, IL-11) that counterbalance innate mucosal immune activation, as it appears to be the case for IL-37.

An additional point to note is that the results reported in Gunaltay et al. [6] are consistent and in line with this group's prior study, describing reduced IL-37 expression and increased chemokine concentrations in colonic biopsies from patients affected by microscopic colitides [8]. Microscopic colitides (MC) including collagenous colitis and lymphocytic colitis feature inappropriate immune activation of the colonic mucosa, characterized by rich lymphocytic infiltrates within the lamina propria and intraepithelial space, with a predominance of cytotoxic $\mathrm{CD}^{+}$cells, and myeloid cells, such as eosinophils and macrophages [9]. Currently, the pathogenesis and etiology of MC are still not completely understood. The present study performed by Gunaltay et al. [6] puts forth an intriguing hypothesis in that, whatever the etiology may be, a general reduction of epithelial-derived IL-37 drives epithelial cells themselves to respond in an exaggerated manner to components of the gut microbiome, specifically TLR5 ligands, by producing an overabundance of proinflammatory molecules. Eventually, this cascade of events triggers and/or perpetuates a detrimental mucosal immune activation and contributes to the specific pathogenesis of MC.

It is also important to consider that studies reporting IL37 expression in the colonic mucosa of IBD patients are conflicting. Ulcerative colitis and Crohn's disease, the two main idiopathic forms of IBD, are chronic, relapsing inflammatory conditions of the gut, characterized by acute and chronic immune cell infiltrates in the epithelium [7]. In the previously cited manuscript from Gunaltay et al. [8], IL-37 was decreased during active ulcerative colitis and upregulated when disease was quiescent, consistent with its anti-inflammatory activities. Nevertheless, studies performed in a pediatric IBD patient population reported intense immunostaining for IL-37 localized to epithelial cells and lymphoid infiltrates in the gut mucosa of affected individuals, and increased IL-37 mRNA transcripts, which together, correlated with disease severity [10]. Yet another study showed elevated IL-37 concentrations, detected by flow cytometry, in circulating B cells, natural killer lymphocytes, and monocytes from adult IBD patients [11]. These discrepancies may not be surprising, considering the different patient populations (adult vs. pediatric), cell types (epithelial vs. immune cells; mucosal vs. circulating immune cells), and disease status (active vs. quiescent) evaluated in these studies.

Finally, and to add further complexity to the understanding of IBD pathogenesis, IL-37 likely exerts its biological effects through two different mechanisms. Il-37 can bind to IL-18R $\alpha$, subsequently dimerizing with IL-1R8/ SIGIRR (as opposed to IL-18R $\beta$ that initiates IL-18 signaling); this IL-18R $\alpha / \mathrm{IL}-1 \mathrm{R} 8$ complex then recruits and inhibits the proinflammatory adaptor protein, MyD88, preventing the activation of downstream NF- $\kappa B-d e p e n d e n t$ events [12]. Alternatively, (and similar to IL-1 $\alpha$ and IL-33), IL-37 may serve as an intracellular nuclear factor, directly binding to DNA elements and downregulating transcription of proinflammatory mediators. In order for nuclear translocation to occur, IL-37 requires the presence of phosphorylatedSMAD3 protein [2]. Importantly, IBD patients usually have elevated gut mucosal levels of SMAD7 at initial evaluation [13], which block the anti-inflammatory activities of transforming growth factor (TGF) $\beta$ through potent inhibition of SMAD3 phosphorylation [14]. A recent phase II trial using an antisense oligonucleotide that blocks SMAD7 revealed a significant clinical benefit in IBD patients, demonstrating the importance of SMAD7 in the pathogenesis of IBD [15]. Therefore, similarly to TGF $\beta$, although IL-37 may be highly expressed in IBD, its anti-inflammatory activity may be prevented due to elevated concentrations of SMAD7 in the local intestinal mucosa of IBD patients. Future studies in this area of investigation are warranted since the extent of SMAD7-mediated blockade of IL-37 is still unknown, 


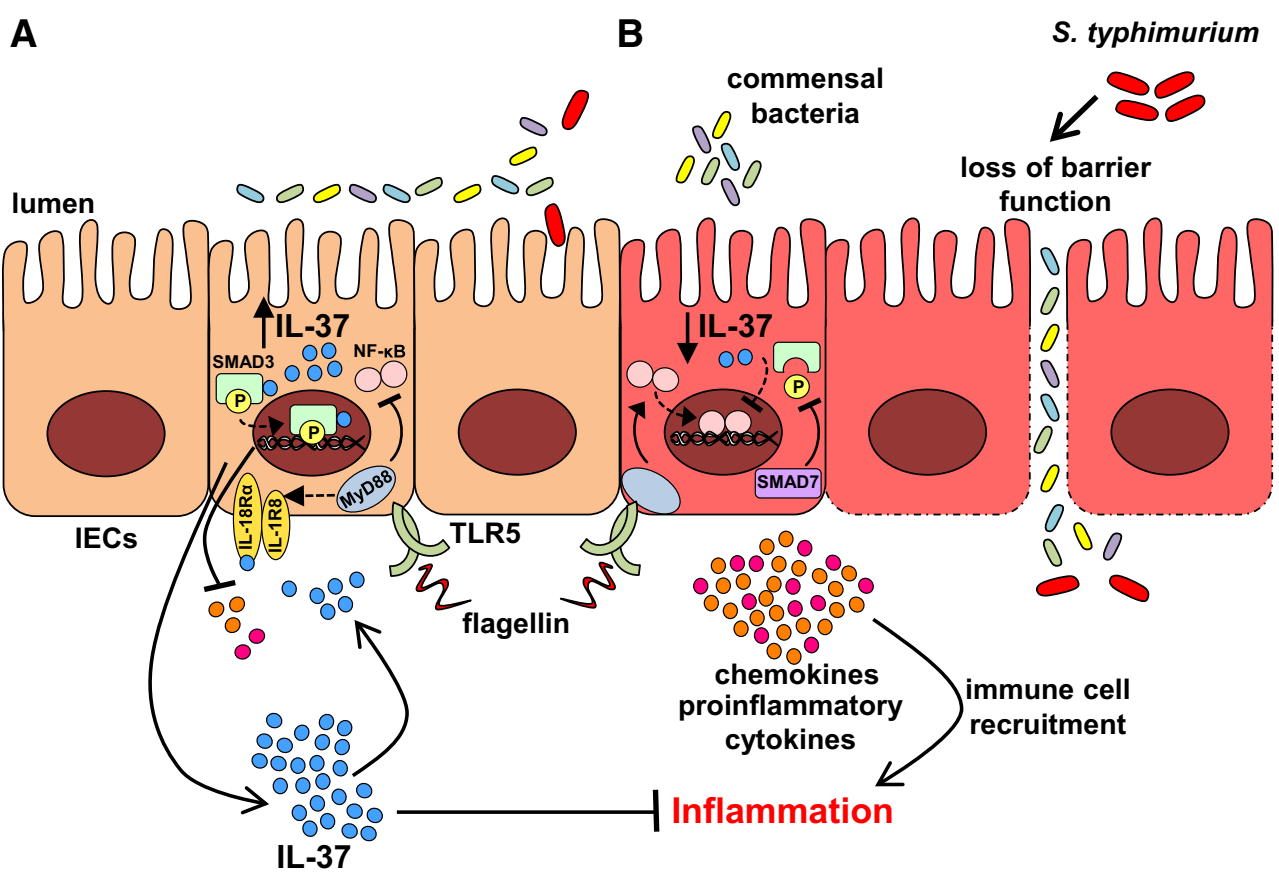

Fig. 1 Working hypothesis of the functions of epithelial-derived IL37 during chronic intestinal inflammation. Intestinal epithelial cells (IEC) sense commensal bacteria or pathogens, which can penetrate the gut barrier, especially during pathogenic disease states, and gain access to basolaterally expressed innate, Toll-like receptors (TLRs), including TLR5, which recognize bacterial components, such as flagellin. TLR5-flagellin engagement activates the adapter protein, MyD88, triggering NF- $\kappa B$ nuclear translocation and downstream production of chemokines and other proinflammatory cytokines. $a$ During homeostatic conditions, healthy IEC express high levels of intracellular IL-37 that bind to phosphorylated $(P)$-SMAD3 and translocate to the nucleus, inhibiting the production of chemokines and proinflammatory cytokines. Secreted IL-37 can also bind to IL$18 \mathrm{R} \alpha$, recruiting IL-1R8/SIGGR to form the IL-18R $\alpha / \mathrm{IL}-1 \mathrm{R} 8$ dimer, which sequesters MyD88 and prevents its activation. $b$ When IL-37 levels are reduced, $\mathrm{NF}-\kappa \mathrm{B}$ is free to translocate to the nucleus; alternatively, increased SMAD7 inhibits SMAD3 phosphorylation, which may prevent subsequent IL-37/SMAD3 nuclear translocation. In both circumstances, production of chemokines and proinflammatory cytokines is no longer inhibited. The result is uncontrolled recruitment and activation of innate immune cells with subsequent amplification of adaptive immune responses leading to chronic intestinal inflammation, such as that observed in MC and IBD particularly regarding the relative contribution of IL-18R $\alpha /$ IL-1R8- and phospho-SMAD3-mediated modulation of IL37 activity.

\section{Conclusion}

The manuscript by Gunaltay and colleagues published in the current issue of Digestive Diseases and Sciences provides new insight into the potential mechanism(s) of chronic inflammatory conditions of the GI tract, such as MC and IBD. In particular, the presence of IL-37, apparently central to the regulation of intestinal immune homeostasis at the epithelial barrier by responding to bacterial sensing specifically through TLR5, may be required to prevent dysregulation of normal gut immunity (Fig. 1). Indeed, most IL-1 family members when produced by the epithelium (vs. immune cells) during early innate events possess protective functions in the regulation of the interplay between the host and the gut microbiome $[1,7]$. In individuals unable to mount appropriate immune responses against harmful factors, perhaps due to deficits in IL-37 production, a homeostatic imbalance can ensue that promotes chronic intestinal inflammation (Fig. 1).

Still, many issues need to be addressed in order to further define the precise function of IL-37 in the GI tract. The lack of a murine homologue will certainly challenge scientists to use innovative approaches and models other than those traditionally used to dissect the in vivo function of other pro-/anti-inflammatory molecules and IL-1 family members. Furthermore, the relative importance of the two different mechanisms of action of IL-37 should also be clarified. Finally, further identification of specific endogenous and exogenous factors able to regulate IL-37 expression in diverse cell types within the intestinal mucosa is of particular relevance in order to better understand the etiopathogenetic processes of MC and IBD. 


\section{Compliance with ethical standards}

Conflict of interest The authors declare no financial arrangements related to the preparation of this manuscript.

\section{References}

1. Lopetuso LR, Chowdhry S, Pizarro TT. Opposing functions of classic and novel IL-1 family members in gut health and disease. Front Immunol. 2013;4:181.

2. Nold MF, Nold-Petry CA, Zepp JA, Palmer BE, Bufler P, Dinarello CA. IL-37 is a fundamental inhibitor of innate immunity. Nat Immunol. 2010;11:1014-1022.

3. Dinarello CA, Nold-Petry C, Nold M et al. Suppression of innate inflammation and immunity by interleukin-37 Eur J Immunol. 2016;46:1067-1081.

4. McNamee EN, Masterson JC, Jedlicka $P$ et al. Interleukin 37 expression protects mice from colitis. Proc Natl Acad Sci USA. 2011;108:16711-16716.

5. Wang WQ, Dong K, Zhou L et al. IL-37b gene transfer enhances the therapeutic efficacy of mesenchumal stromal cells in DSSinduced colitis mice. Acta Pharmacol Sin. 2015;36:1377-1387.

6. Gunaltay S, Ghiboub M, Hultgren O, Hornquist EH. Reduced IL37 production increases spontaneous chemokine expressions in colon epithelial cells. Dig Dis Sci. (Epub ahead of print). doi:10. 1007/s10620-016-4422-9.

7. Pastorelli L, De Salvo C, Mercado JR, Vecchi M, Pizarro TT. Central role of the gut epithelial barrier in the pathogenesis of chronic intestinal inflammation: lessons learned from animal models and human genetics. Front Immunol. 2013;4:280.
8. Gunaltay S, Nyhlin N, Kumawat AK et al. Differential expression of interleukin-1/Toll-like receptor signaling regulators in microscopic and ulcerative colitis World J Gastroenterol. 2014;20: 12249-12259.

9. Pisani LF, Tontini GE, Vecchi M, Pastorelli L. Microscopic colitis: what do we know about pathogenesis? Inflamm Bowel Dis. 2016;22:450-458.

10. Weidlich S, Bulau AM, Schwerd T et al. Intestinal expression of the anti-inflammatory interleukin-1 homologue IL-37 in pediatric inflammatory bowel disease. J Pediatr Gastroenterol Nutr. 2014;59:e18-e26.

11. Fonseca-Camarillo G, Furuzawa-Carballeda J, Yamamoto-Furusho JK. Interleukin 35 (IL-35) and IL-37: Intestinal and peripheral expression by $\mathrm{T}$ and $\mathrm{B}$ regulatory cells in patients with Inflammatory Bowel Disease. Cytokine. 2015;75:389-402.

12. Nold-Petry CA, Lo CY, Rudloff I et al. IL-37 requires the receptors IL-18Ralpha and IL-1R8 (SIGIRR) to carry out its multifaceted anti-inflammatory program upon innate signal transduction. Nat Immunol. 2015;16:354-365.

13. Monteleone G, Kumberova A, Croft NM, McKenzie C, Steer HW, MacDonald TT. Blocking Smad7 restores TGF-beta1 signaling in chronic inflammatory bowel disease. J Clin Investig. 2001;108:601-609.

14. Nakao A, Afrakhte M, Moren A et al. Identification of Smad7, a TGFbeta-inducible antagonist of TGF-beta signalling. Nature. 1997;389:631-635.

15. Monteleone G, Neurath MF, Ardizzone S et al. Mongersen, an oral SMAD7 antisense oligonucleotide, and Crohn's disease. $N$ Engl J Med. 2015;372:1104-1113. 